\title{
POLLEN PROFILE OF PROPOLIS PRODUCED ON THE EASTERN EDGE OF THE SONORAN DESERT IN CENTRAL SONORA, MEXICO
}

\author{
Rey David Vargas-Sánchez ${ }^{1}$, María Cristina Peñalba-Garmendia 2, José Jesús Sánchez- \\ Escalante ${ }^{2}$, Gastón Ramón Torrescano-Urrutia ${ }^{1}$ and Armida SÁnchez-Escalante” ${ }^{1,3}$
}

${ }^{1}$ Centro de Investigación en Alimentación y Desarrollo, A.C. (CIAD), carretera a la Victoria km 0.6, 83000 Hermosillo, Sonora, México. ${ }^{2}$ Universidad de Sonora (UNISON), Blvd. Luis Encinas y Rosales s/n, 83000 Hermosillo, Sonora, México.

${ }^{3}$ Autor para la correspondencia: armida-sanchez@ciad.mx

\begin{abstract}
Eight propolis samples from the eastern edge of the Sonoran Desert were collected at two sites (Pueblo de Álamos and Rancho Viejo, located at Ures, Sonora, Mexico) during two seasons of the year (winter and summer, 2012 and 2013) and analyzed using palynological methods in order to determine which plants were visited by bees in order to collect raw material (resins, wax and pollen) to form propolis. The pollen analysis identified a total of 42 pollen types. Six samples of propolis were bifloral (summer and winter samples) and two multifloral (winter). Mimosa distachya var. laxiflora and Prosopis velutina were considered characteristic pollen types, composing 15 to $45 \%$ of total pollen sums.
\end{abstract}

Key words: botanical origin, pollen analysis, propolis, Sonora-Mexico.

\section{RESUMEN}

Se colectaron ocho muestras de propóleos de la región oriental del Desierto Sonorense en dos sitios (Pueblo de Álamos y Rancho Viejo, ubicados en Ures, Sonora, México) durante dos épocas del año (invierno y verano, 2012 y 2013) y fueron analizadas utilizando métodos palinológicos con el fin de determinar qué plantas fueron visitadas por las abejas para recolectar materia prima (resina, cera y polen) para formar los propóleos. El análisis polínico identificó un total de 42 tipos de polen. Seis muestras de propóleos fueron biflorales (muestras de verano e invierno) y dos multiflorales (muestras de invierno). 
Mimosa distachya var. laxiflora y Prosopis velutina fueron considerados los tipos polínicos característicos, con 15 a $45 \%$ de los conjuntos polínicos totales.

Palabras clave: análisis de polen, origen botánico, propóleos, Sonora-México.

\section{INTRODUCTION}

Propolis, a resinous and adhesive natural substance produced by honeybees (Apis mellifera), has been used most recently as a health food and additive in functional foods, although it has served as a folk medicine for thousands of years. Previous studies have shown that propolis may have numerous beneficial attributes, including antioxidant, antibacterial, antiviral, antifungal and anticancer properties (Farré et al., 2004; Vargas-Sánchez et al., 2013). The different properties were mainly attributed to phytochemicals such as flavonoids and phenolic acids, which form the major components of resin found in propolis samples, where overall, propolis is composed of resins $(50 \%)$, waxes $(30 \%)$, volatile essential oils $(10 \%)$, organic and inorganic chemical compounds (5\%) and pollen (5\%) (Farré et al., 2004). It is well accepted that the chemical composition and properties of propolis greatly depend on the species of honeybee, season, method of harvesting and type of vegetation present in the geographical region (Farré et al., 2004; Peña, 2008). Therefore, it is important to understand by means of pollen identification which species are important for the production of propolis in beekeeping, as this can potentially contribute to a more effective distribution of beehives (Santos, 2011; Silva et al., 2013).

The presence of pollen in sediments from Polish propolis was reported by Warakomska and Maciejewicz (1992), who found that the most abundantly identified pollen came from plants of the Brassicaceae family, mainly Brassica, Sinapis and Raphanus, amounting to $9-33 \%$ of the total pollen content. Salix pollen was present in all samples (6-16\%), including lesser amounts of pollen from Centaurea and Trifolium pratense. Montenegro et al. (2004) identified various kinds of Chilean propolis based on botanical sourcing, including propolis from Escallonia pulverulenta, Nothofagus dombeyi, Mentha pulegium, Eucalyptus and Salix, among other pollen types. In another investigation in Brazil, Barth and Pinto da Luz (2009) evaluated the presence of pollen grains in seven samples of Brazilian red propolis, collected from the northeastern coastal region of Brazil. The presence of Schinus pollen grains was characteristic of the reddish propolis sediments. Recently, Matos et al. (2014) determined the pollen spectrum and levels of total phenolic compounds 
in propolis samples produced by Apis mellifera L. (Apidae), collected from Bahia, Brazil. They found 59 pollen types in the samples, with the Fabaceae family being the most representative. Therefore, the aforementioned studies were able to characterize the regional vegetation from which the propolis was obtained.

The first investigation of Sonoran propolis (Velázquez et al. 2007) addressed the antibacterial (minimum inhibitory concentration, MIC) and antioxidant (freeradical scavenging, FRS) activities of propolis collected from three different areas. Propolis extracts showed strong antibacterial (mainly gram-positive bacteria at 100 $\mu \mathrm{g} / \mathrm{mL})$ and antioxidant activity $(>80 \%$ at $100 \mu \mathrm{g} / \mathrm{mL})$, which were mainly attributed to the presence of flavonoids. Therefore, the authors recommended this natural bee product for use in clinical applications. Sánchez-Escalante et al. (2009) evaluated the antioxidant effect of propolis (produced in the same geographical area) in beef patties, used as a natural preservative. The results suggest that propolis is a strong natural antioxidant, which can be used in fresh meat products to extend shelf life. There are numerous reports in the literature that indicate that active compounds in propolis (mainly organic acids and flavonoids) have antioxidant and antimicrobial properties due to their ability to sequester free radicals, act as substrate for radicals such as superoxide and hydroxyl as well as perform metallic ion chelation. Specifically, the antimicrobial mechanism involves the inhibition of nucleic acids (DNA and RNA) and the degradation of cytoplasmic membranes (Vargas-Sánchez et al., 2014).

However, no detailed studies of pollen analysis exist for the region of Sonora. The aim of this study was to evaluate the pollen composition of Sonoran propolis obtained from the central Sonoran apicultural production area (Ures, Sonora) and to determine its relationship with the regional vegetation and the effect of seasonality on frequency and amount of pollen loads.

\section{MATERIAL AND METHODS}

Samples and study sites

Propolis samples were collected during two seasons of the year: winter (W; propolis samples from the period of August 2012 - March 2013) and summer (S; propolis samples from the March 2013 to August 2013 period). The sampling sites were located at "Pueblo de Álamos" (PA; 298'51.36" N, 1107'26" W; 636 m a.s.1.) and "Rancho Viejo" (RV, 29 $7^{\circ} 19.72 " \mathrm{~N}, 110^{\circ} 16^{\prime} 58.35^{\prime}$ W; $476 \mathrm{~m}$ a.s.l.) within the Ures municipality of central Sonora, Mexico. Both localities present desert climate characteristics, although in terms of annual rainfall, the local climate may be de- 
fined as semi-arid (INEGI, 2012). Annual average temperature and rainfall oscillate from 20 to $22{ }^{\circ} \mathrm{C}$ and 400 to $500 \mathrm{~mm}$ respectively. Seasonality is pronounced and defined by the dominant summer monsoon precipitations (Brito-Castillo et al., 2010; INEGI, 2012; Servicio Meteorológico Nacional, 2012, 2013). Sampling sites are surrounded by foothills of thornscrub, dominated by Fabaceae species (Martínez-Yrízar et al., 2010). Eight propolis samples (PAW12, PAW13, PAS12, PAS13, RVW12, RVW13, RVS12, RVS13) were collected from 15 hives per sampling site during four consecutive seasons. Vegetation surveys were conducted in different seasons within $300 \mathrm{~m}$ of each propolis collection site in order to form a list of plants. The flowering seasons of the taxa of the plant list were determined based on published references (Felger et al., 2001, 2013; Hodgson, 2001; Turner et al., 2005).

Botanical nomenclature

Plant classification follows SEINet thesaurus (2015).

Palynological analysis

For pollen extraction, propolis samples $(0.5 \mathrm{~g})$ were diluted with $1 \mathrm{~mL}$ ethanol and centrifuged at $4000 \mathrm{rpm}$ for $10 \mathrm{~min}$. After cleaning the samples, pollen grains were further dehydrated in glacial acetic acid and prepared for melissopalynological analysis using the acetolysis method (Erdtman, 1960). This consisted of boiling in a water bath $\left(92{ }^{\circ} \mathrm{C} / 10 \mathrm{~min}\right)$ with a mixture of acetic anhydride - sulphuric acid (9:1) and subsequently centrifuging and decanting the supernatant. Slides were mounted with glycerin and sealed with nail polish.

All samples were observed using an optical microscope (Olympus CX31) at x200, x400 and x1000. To determine the frequency classes, 500 pollen grains were counted from each sample. Pollen types were assigned to four percentage classes, as determined by Barth (2004): predominant pollen ( $>45 \%$ ), secondary pollen (15 to $45 \%$ ), important minor pollen ( 3 to $15 \%$ ) and minor pollen ( $<3 \%$ ). Pollen slides from the pollen reference collection of the Department of Scientific and Technological Research, Sonora University (UNISON) in Sonora, Mexico and published a pollen atlas (Roubik and Moreno, 1991) were used to identify the pollen grains, in addition to the identification of pollen grains based on plant species collected from the sampling areas.

Statistical analysis

All data were submitted to an analysis of variance (ANOVA) with a posthoc determination using Tukey's test. A Detrended correspondence analysis 
(DCA) and a Pearson's correlation coefficient $(r)$ were applied to examine the correlation between all the variables. The level of significance was set at $P$ $<0.05$ (Software package, SPSS version 21; and PAST-Palaeontological statistics version 3.07).

\section{RESULTS}

Botanical origin of central Sonoran propolis

The vegetation of the sampling zones was dominated by subtropical species (Table 1), mainly from the Fabaceae (18.5\%), Cactaceae (10.2\%), Malvaceae (8.3\%) and Asteraceae (6.5\%) families. Pollen analysis showed a diversity of pollen types in the studied propolis samples. A total of 42 pollen types (Table 2) belonging to 22 families were identified; some of the principal pollen types are illustrated in Fig. 1. The families with the highest pollen frequencies $(>15 \%,<45 \%)$ were Fabaceae, Asteraceae, Burseraceae, Chenopodiaceae-Amaranthaceae and Zygophyllaceae. The highest pollen concentrations were identified in the summer propolis samples, corresponding to Mimosa distachya var. laxiflora (PAS12, 37\%; PAS13, 41.6\%) in Pueblo de Álamos and Prosopis velutina (RVS12, 23.1\%; RVS13, 29.2\%) in Rancho Viejo. A reduction in the frequencies of these pollen types was found for propolis samples produced during the winter, both at Pueblo de Álamos (Mimosa distachya var. laxiflora; PAW12, 18.7\%; PAW13, 19.4\%) and Rancho Viejo (Prosopis velutina; RVW12, 13.3\%; RVW13, 16.7\%). According to these samples, six bifloral (15 to $45 \%$ pollen frequencies: PAW12, PAS12, PAS13, RVW13, RVS12 and RVS13) and two multifloral $(<15 \%$ pollen frequencies: RVW12 and PAW13) propolis samples were recorded, distributed among both regions.

Relationship between propolis samples of different origin

In order to evaluate the differences among propolis samples collected from different sites and seasons, a multivariate analysis (detrended correspondence analyses, DCA) was performed (Fig. 2). The ordination of samples and pollen source based on the two first axes (Axis 1 and Axis 2) of the DCA indicate that analyzed propolis were separated seasonally (summer samples left direction and winter samples clockwise), and slightly geographically. Most pollen types are clearly grouped by families and Prosopis pollen was significantly closer to propolis samples collected in both study sites $(P<0.05)$. Also, propolis samples from PA are clearly more grouped at the top left than RV, mainly in summer. As a result, 
Table 1. Common plant taxa in the areas surrounding the study sites and their flowering seasons.

\begin{tabular}{|c|c|c|}
\hline Acanthaceae & $\begin{array}{l}\text { Dicliptera } \mathrm{sp} .{ }^{(\mathrm{Ns} ; \mathrm{PA}, \mathrm{RV})} \\
\text { Elytraria imbricata }^{(\mathrm{S} ; \mathrm{PA})} \\
\text { Henrya insularis }^{(\mathrm{Sp} ; \mathrm{PA})}\end{array}$ & $\begin{array}{l}\text { Justicia candicans }^{(\mathrm{Sp}, \mathrm{F}, \mathrm{W} ; \mathrm{PA})} \\
\text { Tetramerium nervosum }^{(\mathrm{F}, \mathrm{W}, \mathrm{Sp} ; \mathrm{RV})}\end{array}$ \\
\hline Achatocarpaceae & Phaulothamnus spinescens (S, F; PA) & \\
\hline Agavaceae & Agave angustifolia ${ }^{(\mathrm{W}, \mathrm{Sp} ; \mathrm{PA})}$ & \\
\hline Asteraceae & $\begin{array}{l}\text { Ambrosia }^{(\mathrm{Sp} ; \mathrm{PA}, \mathrm{RV})} \\
\text { Artemisia }^{(\mathrm{Ns} ; \mathrm{RV})} \\
\text { Brickellia } \text { coulteri }^{(\mathrm{Sp}, \mathrm{S}, \mathrm{F} ; \mathrm{PA}, \mathrm{RV})}\end{array}$ & $\begin{array}{l}\text { Lagascea decipiens }^{(\mathrm{F}, \mathrm{W}, \mathrm{Sp} ; \mathrm{PA})} \\
\text { Malacothrix sonorae }^{(\mathrm{W}, \mathrm{Sp} ; \mathrm{PA})} \\
\text { Perityle reinana }^{(\mathrm{Ns} ; \mathrm{PA})}\end{array}$ \\
\hline Boraginaceae & $\begin{array}{l}\text { Cordia sonorae }^{(\mathrm{Sp} ; \mathrm{PA})} \\
\text { Nama hispidum }^{(\mathrm{Sp} ; \mathrm{PA}, \mathrm{RV})}\end{array}$ & Phacelia gentryi (Sp, S, F, W; PA, RV) \\
\hline Brassicaceae & Descurainia pinnata $^{(\mathrm{W}, \mathrm{Sp} ; \mathrm{PA})}$ & \\
\hline Burseraceae & $\begin{array}{l}\text { Bursera fagaroides }^{(\mathrm{S} ; \mathrm{PA})} \\
\text { Bursera laxiflora }^{(\mathrm{F}, \mathrm{W} ; \mathrm{PA}, \mathrm{RV})}\end{array}$ & Bursera microphylla ${ }^{(\mathrm{S} ; \mathrm{PA}, \mathrm{RV})}$ \\
\hline Cactaceae & $\begin{array}{l}\text { Cylindropuntia fulgida }{ }^{(\mathrm{S} ; \mathrm{PA})} \\
\text { Cylindropuntia leptocaulis }^{(\mathrm{Sp}, \mathrm{S} ; \mathrm{PA}, \mathrm{RV})} \\
\text { Cylindropuntia thurberi }^{(\mathrm{Ns} ; \mathrm{PA}, \mathrm{RV})} \\
\text { Mammillaria standleyi }^{(\mathrm{S} ; \mathrm{PA})} \\
\text { Opuntia engelmannii }^{(\mathrm{Sp}, \mathrm{S} ; \mathrm{PA}, \mathrm{RV})} \\
\text { Opuntia pubescens }^{(\mathrm{Ns} ; \mathrm{PA})}\end{array}$ & $\begin{array}{l}\text { Opuntia } \text { sp. }{ }^{(\mathrm{Ns} ; \mathrm{PA})} \\
\text { Pachycereus pecten-aboriginum } \\
\quad \text { (Sp, S, F, W; PA, RV) } \\
\text { Stenocereus alamosensis }{ }^{(\mathrm{Ns} ; \mathrm{PA}, \mathrm{RV})} \\
\text { Stenocereus thurberi }{ }^{(\mathrm{S} ; \mathrm{PA}, \mathrm{RV})}\end{array}$ \\
\hline Cannabaceae & $\begin{array}{l}\text { Celtis pallida }^{(\mathrm{Sp}, \mathrm{S}, \mathrm{F}, \mathrm{W} ; \mathrm{PA}, \mathrm{RV})} \\
\text { Celtis reticulata }^{(\mathrm{Sp}, \mathrm{S} ; \mathrm{PA}, \mathrm{RV})}\end{array}$ & \\
\hline Chenopodiaceae & Chenopodium sp. ${ }^{(\mathrm{Ns} ; \mathrm{PA}, \mathrm{RV})}$ & \\
\hline Convolvulaceae & $\begin{array}{l}\text { Cuscuta sp. }{ }^{(\mathrm{Ns} ; \mathrm{PA})} \\
\text { Evolvulus alsinoides } \text { (Sp, S, F, W; PA) }^{-}\end{array}$ & Ipomoea arborescens ${ }^{(\mathrm{F}, \mathrm{W} ; \mathrm{PA}, \mathrm{RV})}$ \\
\hline Euphorbiaceae & $\begin{array}{l}\text { Jatropha cardiophylla }{ }^{(\mathrm{S} ; \mathrm{RV})} \\
\text { Jatropha cordata }^{(\mathrm{F} ; \mathrm{PA}, \mathrm{RV})}\end{array}$ & Tragia $\mathrm{sp} .{ }^{(\mathrm{Ns} ; \mathrm{PA})}$ \\
\hline Fabaceae & 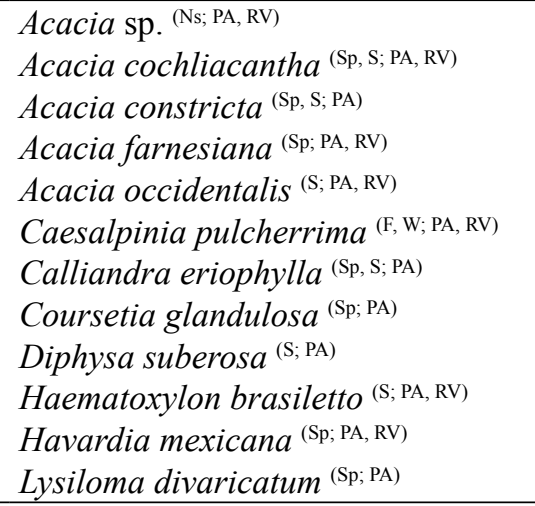 & 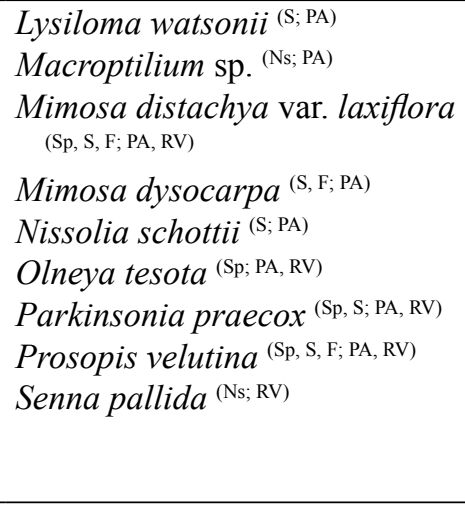 \\
\hline Fouquieriaceae & $\begin{array}{l}\text { Fouquieria macdougalii }(\mathrm{Sp}, \mathrm{S} ; \mathrm{PA}, \mathrm{RV}) \\
\text { Fouquieria splendens }{ }_{(\mathrm{Sp} ; \mathrm{PA}, \mathrm{RV})}\end{array}$ & \\
\hline
\end{tabular}


Table 1. Continued.

\begin{tabular}{|c|c|c|}
\hline Lamiaceae & Hyptis albida ${ }^{(\mathrm{Sp}, \mathrm{F}, \mathrm{W} ; \mathrm{PA}, \mathrm{RV})}$ & \\
\hline Malpighiaceae & Callaeum macropterum (Sp, S, F, W; PA, RV) & \\
\hline Malvaceae & 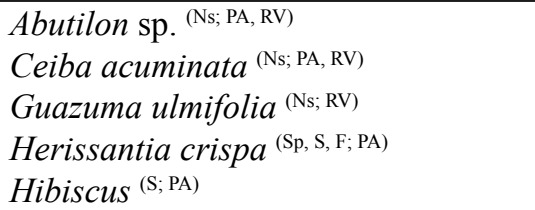 & $\begin{array}{l}\text { Sida alamosana }{ }^{(\mathrm{Ns} ; \mathrm{PA}, \mathrm{RV})} \\
\text { Sphaeralcea } \mathrm{sp} .{ }^{(\mathrm{Sp} ; \mathrm{RV})} \\
\text { Waltheria } \mathrm{sp} .{ }^{(\mathrm{Ns} ; \mathrm{PA})}\end{array}$ \\
\hline Myrtaceae & Eucalyptus $\mathrm{sp} .{ }^{(\mathrm{Ns} ; \mathrm{PA}, \mathrm{RV})}$ & \\
\hline Nyctaginaceae & Nyctaginaceae sp. ${ }^{(\mathrm{Ns} ; \mathrm{PA}, \mathrm{RV})}$ & \\
\hline Onagraceae & Oenothera sp. $^{(\mathrm{Ne} ; \mathrm{PA})}$ & \\
\hline Papaveraceae & Eschscholzia californica ${ }^{(\mathrm{S} ; \mathrm{PA})}$ & \\
\hline Poaceae & $\begin{array}{l}\text { Erioneuron pulchellum }{ }^{(\mathrm{S} ; \mathrm{PA}, \mathrm{RV})} \\
\text { Melinis repens }{ }^{(\mathrm{Ns} ; \mathrm{PA}, \mathrm{RV})}\end{array}$ & Setaria sp. $^{(\mathrm{Ns} ; \mathrm{PA}, \mathrm{RV})}$ \\
\hline Rhamnaceae & Karwinskia humboldtiana ${ }^{(\mathrm{S} ; \mathrm{PA})}$ & \\
\hline Rubiaceae & $\begin{array}{l}\text { Hintonia latiflora }^{(\mathrm{Ns} ; \mathrm{PA})} \\
\text { Mitracarpus hirtus }^{(\mathrm{Ns} ; \mathrm{PA})}\end{array}$ & Randia obcordata (S; $\mathrm{PA}, \mathrm{RV})$ \\
\hline Rutaceae & Zanthoxylum fagara ${ }^{(\mathrm{S}, \mathrm{F} ; \mathrm{PA})}$ & \\
\hline Sapindaceae & $\begin{array}{l}\text { Cardiospermum halicacabum } \\
\mathrm{RV})\end{array}$ & \\
\hline Sapotaceae & Sideroxylon occidentale ${ }^{(\mathrm{S}, \mathrm{F}, \mathrm{W} ; \mathrm{PA})}$ & \\
\hline Solanaceae & $\begin{array}{l}\text { Capsicum annuum } \text { var. } \\
\text { glabriusculum }^{(\mathrm{Ns} ; \mathrm{PA})} \\
\text { Lycium andersonii }^{(\mathrm{F}, \mathrm{W} ; \mathrm{PA})} \text {, }\end{array}$ & $\begin{array}{l}\text { Lycium berlandieri }^{(\mathrm{F}, \mathrm{W} ; \mathrm{PA})} \\
\text { Physalis } \mathrm{sp} \text {. }^{\mathrm{Ns} ; \mathrm{PA})} \\
\text { Solanum tridynamum }\left({ }^{(\mathrm{Sp}, \mathrm{S} ; \mathrm{PA})}\right.\end{array}$ \\
\hline Scrophulariaceae & $\begin{array}{l}\text { Mimulus floribundus }{ }^{(\mathrm{Sp}, \mathrm{S}, \mathrm{F} ; \mathrm{PA})} \\
\text { Stemodia durantifolia }^{(\mathrm{Sp}, \mathrm{S}, \mathrm{F}, \mathrm{W} ; \mathrm{PA})}\end{array}$ & \\
\hline Verbenaceae & Aloysia gratissima $^{(\mathrm{Ns} ; \mathrm{PA})}$ & Vitex mollis ${ }^{(\mathrm{Ns} ; \mathrm{PA})}$ \\
\hline Viscaceae & Phoradendron californicum ${ }^{(\mathrm{W} ; \mathrm{PA})}$ & \\
\hline Zygophyllaceae & Guaiacum coulteri $(\mathrm{S} ; \mathrm{PA}, \mathrm{RV})$ & \\
\hline Pteridaceae & Cheilanthes pringlei $^{(\mathrm{Ns} ; \mathrm{PA})}$ & \\
\hline
\end{tabular}

Seasons (Spring, Sp; Summer, S; Fall, F; Winter, W; Ns, not specified). Study areas (Pueblo of Álamos, PA; Rancho Viejo, RV).

this was also observed for summer propolis samples from PA (Table 2), which showed high levels of Mimosa, while summer propolis from RV showed a high content of Prosopis pollen $(P<0.05)$, which corresponds to differences in the local vegetation (Mimosa is more abundant in the thornscrub foothills of PA and 
Acta Botanica Mexicana 114: 69-86 (2016)

Table 2. Frequencies of pollen types identified in propolis samples.

\begin{tabular}{|c|c|c|c|c|c|c|c|c|}
\hline Pollen types & PAW12 & PAW13 & PAS12 & PAS13 & RVW12 & RVW13 & RVS12 & RVS13 \\
\hline \multicolumn{9}{|l|}{ Acanthaceae } \\
\hline Dicliptera & $4.0^{\mathrm{bB}}$ & $3.2^{\mathrm{aB}}$ & $0.7^{\mathrm{bA}}$ & $0.0^{\mathrm{aA}}$ & $1.1^{\mathrm{bB}}$ & $0.3^{\mathrm{aA}}$ & $0.4^{\mathrm{bA}}$ & $3.9^{\mathrm{aB}}$ \\
\hline \multicolumn{9}{|l|}{ Agavaceae } \\
\hline Agave angustifolia & $0.0^{\mathrm{aA}}$ & $0.0^{\mathrm{aA}}$ & $0.0^{\mathrm{aA}}$ & $0.0^{\mathrm{aA}}$ & $0.0^{\mathrm{aA}}$ & $0.0^{\mathrm{aA}}$ & $0.0^{\mathrm{aA}}$ & $0.2^{\mathrm{bB}}$ \\
\hline \multicolumn{9}{|l|}{ Asteraceae } \\
\hline unknown taxa & $7.0^{\mathrm{aB}}$ & $10.0^{\mathrm{bB}}$ & $3.7^{\mathrm{aA}}$ & $8.6^{\mathrm{bA}}$ & $10.5^{\mathrm{bB}}$ & $9.8^{\mathrm{aB}}$ & $6.9^{\mathrm{bA}}$ & $6.0^{\mathrm{aA}}$ \\
\hline Ambrosia & $5.3^{\mathrm{aB}}$ & $8.7^{\mathrm{bB}}$ & $2.5^{\mathrm{bA}}$ & $0.2^{\mathrm{aA}}$ & $5.5^{\mathrm{bB}}$ & $5.4^{\mathrm{aB}}$ & $2.8^{\mathrm{aA}}$ & $3.5^{\mathrm{bA}}$ \\
\hline Artemisia & $0.0^{\mathrm{aA}}$ & $0.0^{\mathrm{aA}}$ & $0.0^{\mathrm{aA}}$ & $0.0^{\mathrm{aA}}$ & $0.0^{\mathrm{aA}}$ & $0.0^{\mathrm{aA}}$ & $0.2^{\mathrm{bB}}$ & $0.0^{\mathrm{aA}}$ \\
\hline \multicolumn{9}{|l|}{ Boraginaceae } \\
\hline Nama hispidum & $0.0^{\mathrm{aA}}$ & $1.3^{\mathrm{bB}}$ & $0.0^{\mathrm{aA}}$ & $0.0^{\mathrm{aA}}$ & $0.0^{\mathrm{aA}}$ & $0.0^{\mathrm{aA}}$ & $0.2^{\mathrm{aB}}$ & $0.9^{\mathrm{bB}}$ \\
\hline Phacelia gentryi & $0.3^{\mathrm{bB}}$ & $0.0^{\mathrm{aA}}$ & $0.0^{\mathrm{aA}}$ & $0.0^{\mathrm{aA}}$ & $0.0^{\mathrm{aA}}$ & $0.0^{\mathrm{aA}}$ & $2.0^{\mathrm{bB}}$ & $0.0^{\mathrm{aA}}$ \\
\hline \multicolumn{9}{|l|}{ Burseraceae } \\
\hline Bursera fagaroides type & $7.7^{\mathrm{bB}}$ & $4.5^{\mathrm{aB}}$ & $3.5^{\mathrm{bA}}$ & $1.6^{\mathrm{aA}}$ & $5.3^{\mathrm{bA}}$ & $4.5^{\mathrm{aB}}$ & $7.1^{\mathrm{bB}}$ & $2.2^{\mathrm{aA}}$ \\
\hline Bursera laxiflora type & $6.0^{\mathrm{bB}}$ & $4.9^{\mathrm{aB}}$ & $3.5^{\mathrm{bA}}$ & $1.4^{\mathrm{aA}}$ & $10.0^{\mathrm{bB}}$ & $6.3^{\mathrm{aB}}$ & $8.5^{\mathrm{bA}}$ & $3.5^{\mathrm{aA}}$ \\
\hline \multicolumn{9}{|l|}{ Cactaceae } \\
\hline unknown taxa & $4.0^{\mathrm{bB}}$ & $2.3^{\mathrm{aB}}$ & $1.0^{\mathrm{bA}}$ & $0.0^{\mathrm{aA}}$ & $3.3^{\mathrm{bB}}$ & $2.1^{\mathrm{aB}}$ & $1.2^{\mathrm{aA}}$ & $1.7^{\mathrm{bA}}$ \\
\hline Cylindropuntia & $0.0^{\mathrm{aA}}$ & $0.0^{\mathrm{aA}}$ & $0.0^{\mathrm{aA}}$ & $0.0^{\mathrm{aA}}$ & $0.3^{\mathrm{aA}}$ & $0.6^{\mathrm{bB}}$ & $1.0^{\mathrm{bB}}$ & $0.0^{\mathrm{aA}}$ \\
\hline Stenocereus type & $0.0^{\mathrm{aA}}$ & $0.0^{\mathrm{aA}}$ & $0.0^{\mathrm{aA}}$ & $0.0^{\mathrm{aA}}$ & $0.0^{\mathrm{aA}}$ & $0.3^{\mathrm{bB}}$ & $0.0^{\mathrm{aA}}$ & $0.0^{\mathrm{aA}}$ \\
\hline \multicolumn{9}{|l|}{ Cannabaceae } \\
\hline Celtis reticulata & $0.0^{\mathrm{aA}}$ & $0.0^{\mathrm{aA}}$ & $0.0^{\mathrm{aA}}$ & $0.0^{\mathrm{aA}}$ & $0.6^{\mathrm{bB}}$ & $0.0^{\mathrm{aA}}$ & $0.2^{\mathrm{bA}}$ & $0.0^{\mathrm{aA}}$ \\
\hline \multicolumn{9}{|l|}{ Chenop-Amaranthaceae } \\
\hline Chenopodium type & $3.7^{\mathrm{aB}}$ & $8.7^{\mathrm{bB}}$ & $2.7^{\mathrm{bA}}$ & $0.2^{\mathrm{aA}}$ & $11.1^{\mathrm{aB}}$ & $15.2^{\mathrm{bB}}$ & $2.4^{\mathrm{aA}}$ & $3.5^{\mathrm{bA}}$ \\
\hline \multicolumn{9}{|l|}{ Convolvulaceae } \\
\hline Cuscuta & $0.0^{\mathrm{aA}}$ & $0.0^{\mathrm{aA}}$ & $0.0^{\mathrm{aA}}$ & $0.2^{\mathrm{bB}}$ & $0.0^{\mathrm{aA}}$ & $0.0^{\mathrm{aA}}$ & $0.0^{\mathrm{aA}}$ & $0.0^{\mathrm{aA}}$ \\
\hline Ipomoea & $2.3^{\mathrm{bB}}$ & $0.6^{\mathrm{aA}}$ & $1.2^{\mathrm{bA}}$ & $0.7^{\mathrm{aB}}$ & $1.9^{\mathrm{bB}}$ & $1.5^{\mathrm{aB}}$ & $0.4^{\mathrm{aA}}$ & $0.9^{\mathrm{bA}}$ \\
\hline \multicolumn{9}{|l|}{ Fabaceae } \\
\hline Acacia & $5.7^{\mathrm{bA}}$ & $5.5^{\mathrm{aA}}$ & $11.1^{\mathrm{bB}}$ & $6.8^{\mathrm{aB}}$ & $5.5^{\mathrm{bB}}$ & $4.2^{\mathrm{aA}}$ & $4.0^{\mathrm{aA}}$ & $4.8^{\mathrm{bB}}$ \\
\hline Caesalpinia pulcherrima & $1.3^{\mathrm{bA}}$ & $0.3^{\mathrm{aA}}$ & $1.5^{\mathrm{aB}}$ & $11.6^{\mathrm{bB}}$ & $3.3^{\mathrm{bA}}$ & $1.5^{\mathrm{aB}}$ & $3.4^{\mathrm{bB}}$ & $0.9^{\mathrm{aA}}$ \\
\hline Havardia type & $0.7^{\mathrm{bA}}$ & $0.3^{\mathrm{aA}}$ & $2.7^{\mathrm{aB}}$ & $2.8^{\mathrm{bB}}$ & $3.6^{\mathrm{bB}}$ & $2.1^{\mathrm{aB}}$ & $1.8^{\mathrm{bA}}$ & $0.0^{\mathrm{aA}}$ \\
\hline Haematoxylon brasiletto & $0.0^{\mathrm{aA}}$ & $0.3^{\mathrm{bB}}$ & $0.7^{\mathrm{bB}}$ & $0.0^{\mathrm{aA}}$ & $0.8^{\mathrm{aA}}$ & $0.9^{\mathrm{bA}}$ & $2.8^{\mathrm{bB}}$ & $1.3^{\mathrm{aB}}$ \\
\hline Mimosa & $18.7^{\mathrm{aA}}$ & $19.4^{\mathrm{bA}}$ & $37.0^{\mathrm{aB}}$ & $41.6^{\mathrm{bB}}$ & $13.3^{\mathrm{aA}}$ & $16.7^{\mathrm{bA}}$ & $21.9^{\mathrm{aB}}$ & $25.5^{\mathrm{bB}}$ \\
\hline Olneya tesota & $2.0^{\mathrm{bB}}$ & $1.0^{\mathrm{aB}}$ & $0.5^{\mathrm{bA}}$ & $0.0^{\mathrm{aA}}$ & $1.9^{\mathrm{bB}}$ & $1.2^{\mathrm{aA}}$ & $0.0^{\mathrm{aA}}$ & $2.6^{\mathrm{bB}}$ \\
\hline Parkinsonia praecox & $1.0^{\mathrm{aB}}$ & $1.0^{\mathrm{aB}}$ & $0.5^{\mathrm{bA}}$ & $0.0^{\mathrm{aA}}$ & $0.6^{\mathrm{bB}}$ & $0.3^{\mathrm{aA}}$ & $0.0^{\mathrm{aA}}$ & $0.6^{\mathrm{bB}}$ \\
\hline Prosopis velutina & $15.3^{\mathrm{bA}}$ & $13.6^{\mathrm{aA}}$ & $19.8^{\mathrm{bB}}$ & $16.0^{\mathrm{aB}}$ & $15.2^{\mathrm{aA}}$ & $17.3^{\mathrm{bA}}$ & $23.1^{\mathrm{aB}}$ & $29.2^{\mathrm{bB}}$ \\
\hline
\end{tabular}


Table 2. Continued.

\begin{tabular}{|c|c|c|c|c|c|c|c|c|}
\hline Pollen types & PAW12 & PAW13 & PAS12 & PAS13 & RVW12 & RVW13 & RVS12 & RVS13 \\
\hline \multicolumn{9}{|l|}{ Fagaceae } \\
\hline Quercus & $0.0^{\mathrm{aA}}$ & $0.0^{\mathrm{aA}}$ & $0.2^{\mathrm{aB}}$ & $0.4^{\mathrm{bB}}$ & $0.8^{\mathrm{aB}}$ & $1.5^{\mathrm{bB}}$ & $0.0^{\mathrm{aA}}$ & $0.0^{\mathrm{aA}}$ \\
\hline \multicolumn{9}{|l|}{ Fouquieriaceae } \\
\hline Fouquieria & $0.0^{\mathrm{aA}}$ & $0.6^{\mathrm{bA}}$ & $0.2^{\mathrm{aB}}$ & $2.8^{\mathrm{bB}}$ & $0.0^{\mathrm{aA}}$ & $0.0^{\mathrm{aA}}$ & $0.2^{\mathrm{aB}}$ & $0.4^{\mathrm{bB}}$ \\
\hline \multicolumn{9}{|l|}{ Lamiaceae } \\
\hline Hyptis albida & $0.3^{\mathrm{bA}}$ & $0.0^{\mathrm{aA}}$ & $1.5^{\mathrm{bB}}$ & $0.0^{\mathrm{aA}}$ & $0.8^{\mathrm{bB}}$ & $0.3^{\mathrm{aB}}$ & $0.6^{\mathrm{bA}}$ & $0.0^{\mathrm{aA}}$ \\
\hline \multicolumn{9}{|l|}{ Malvaceae } \\
\hline Abutilon & $2.0^{\mathrm{aB}}$ & $2.9^{\mathrm{bB}}$ & $0.0^{\mathrm{aA}}$ & $0.0^{\mathrm{aA}}$ & $1.1^{\mathrm{aB}}$ & $1.5^{\mathrm{bB}}$ & $0.4^{\mathrm{bA}}$ & $0.0^{\mathrm{aA}}$ \\
\hline Ceiba acuminata & $0.0^{\mathrm{aA}}$ & $0.6^{\mathrm{bB}}$ & $0.0^{\mathrm{aA}}$ & $0.0^{\mathrm{aA}}$ & $0.0^{\mathrm{aA}}$ & $0.0^{\mathrm{aA}}$ & $0.0^{\mathrm{aA}}$ & $0.0^{\mathrm{aA}}$ \\
\hline Herissantia & $0.0^{\mathrm{aA}}$ & $2.9^{\mathrm{bB}}$ & $0.0^{\mathrm{aA}}$ & $0.0^{\mathrm{aA}}$ & $0.0^{\mathrm{aA}}$ & $0.0^{\mathrm{aA}}$ & $0.0^{\mathrm{aA}}$ & $0.0^{\mathrm{aA}}$ \\
\hline Hibiscus type & $0.0^{\mathrm{aA}}$ & $0.0^{\mathrm{aA}}$ & $0.0^{\mathrm{aA}}$ & $0.2^{\mathrm{bB}}$ & $0.0^{\mathrm{aA}}$ & $0.0^{\mathrm{aA}}$ & $0.0^{\mathrm{aA}}$ & $0.0^{\mathrm{aA}}$ \\
\hline Sida & $0.0^{\mathrm{aA}}$ & $0.0^{\mathrm{aA}}$ & $0.0^{\mathrm{aA}}$ & $0.2^{\mathrm{bB}}$ & $0.0^{\mathrm{aA}}$ & $0.0^{\mathrm{aA}}$ & $0.2^{\mathrm{bB}}$ & $0.0^{\mathrm{aA}}$ \\
\hline Sphaeralcea & $0.0^{\mathrm{aA}}$ & $0.0^{\mathrm{aA}}$ & $0.0^{\mathrm{aA}}$ & $0.0^{\mathrm{aA}}$ & $0.0^{\mathrm{aA}}$ & $0.0^{\mathrm{aA}}$ & $0.8^{\mathrm{bB}}$ & $0.0^{\mathrm{aA}}$ \\
\hline \multicolumn{9}{|l|}{ Myrtaceae } \\
\hline Eucalyptus & $0.0^{\mathrm{aA}}$ & $1.6^{\mathrm{bB}}$ & $0.7^{\mathrm{aB}}$ & $0.9^{\mathrm{bA}}$ & $1.4^{\mathrm{bB}}$ & $1.2^{\mathrm{aB}}$ & $0.4^{\mathrm{aA}}$ & $0.7^{\mathrm{bA}}$ \\
\hline \multicolumn{9}{|l|}{ Nyctaginaceae } \\
\hline unknown taxa & $0.7^{\mathrm{bB}}$ & $0.0^{\mathrm{aA}}$ & $0.0^{\mathrm{aA}}$ & $0.0^{\mathrm{aA}}$ & $0.3^{\mathrm{aB}}$ & $0.3^{\mathrm{aA}}$ & $0.0^{\mathrm{aA}}$ & $2.2^{\mathrm{bB}}$ \\
\hline \multicolumn{9}{|l|}{ Poaceae } \\
\hline unknown taxa & $2.8^{\mathrm{bB}}$ & $1.6^{\mathrm{aB}}$ & $0.0^{\mathrm{aA}}$ & $0.5^{\mathrm{bA}}$ & $0.3^{\mathrm{aA}}$ & $0.9^{\mathrm{bA}}$ & $2.8^{\mathrm{aB}}$ & $2.6^{\mathrm{bB}}$ \\
\hline \multicolumn{9}{|l|}{ Rhamnaceae } \\
\hline Karwinskia & $1.7^{\mathrm{bB}}$ & $0.6^{\mathrm{aB}}$ & $0.5^{\mathrm{bA}}$ & $0.0^{\mathrm{aA}}$ & $0.0^{\mathrm{a}} \mathrm{A}$ & $0.0^{\mathrm{aA}}$ & $0.0^{\mathrm{aA}}$ & $0.0^{\mathrm{aA}}$ \\
\hline \multicolumn{9}{|l|}{ Rubiaceae } \\
\hline Mitracarpus hirtus & $0.0^{\mathrm{aA}}$ & $0.0^{\mathrm{aA}}$ & $0.0^{\mathrm{aA}}$ & $0.2^{\mathrm{bB}}$ & $0.0^{\mathrm{aA}}$ & $0.0^{\mathrm{aA}}$ & $0.0^{\mathrm{aA}}$ & $0.0^{\mathrm{aA}}$ \\
\hline Randia obcordata & $0.0^{\mathrm{aA}}$ & $0.0^{\mathrm{aA}}$ & $0.0^{\mathrm{aA}}$ & $0.0^{\mathrm{aA}}$ & $0.0^{\mathrm{aA}}$ & $0.0^{\mathrm{aA}}$ & $0.8^{\mathrm{bB}}$ & $0.0^{\mathrm{aA}}$ \\
\hline Randia sp. & $2.7^{\mathrm{bB}}$ & $0.0^{\mathrm{aA}}$ & $0.5^{\mathrm{bA}}$ & $0.0^{\mathrm{aA}}$ & $0.0^{\mathrm{aA}}$ & $0.0^{\mathrm{aA}}$ & $0.0^{\mathrm{aA}}$ & $0.0^{\mathrm{aA}}$ \\
\hline \multicolumn{9}{|l|}{ Sapindaceae } \\
\hline Cardiospermum & $0.7^{\mathrm{bB}}$ & $0.6^{\mathrm{aA}}$ & $0^{\mathrm{aA}}$ & $1.1^{\mathrm{bB}}$ & $0.3^{\mathrm{bA}}$ & $0.0^{\mathrm{aA}}$ & $0.8^{\mathrm{bB}}$ & $0.7^{\mathrm{aB}}$ \\
\hline \multicolumn{9}{|l|}{ Zygophyllaceae } \\
\hline Guaiacum coulteri & $3.7^{\mathrm{bB}}$ & $1.3^{\mathrm{aB}}$ & $1.5^{\mathrm{bA}}$ & $1.1^{\mathrm{aA}}$ & $0.8^{\mathrm{aA}}$ & $2.7^{\mathrm{bB}}$ & $2.4^{\mathrm{bB}}$ & $1.3^{\mathrm{aA}}$ \\
\hline Unidentified & 0.7 & 1.3 & 2.2 & 1.2 & 0.3 & 1.8 & 0.6 & 0.6 \\
\hline Total of pollen types $(\%)$ & 100 & 100 & 100 & 100 & 100 & 100 & 100 & 100 \\
\hline
\end{tabular}

Pueblo de Álamos (PA); Rancho Viejo (RV); winter (W) and summer (S); 2012 (12) and 2013 (13). Different superscripts (a-b) within the same sample and season, and (A-B) within the same year of collection and different season differ significantly $(P<0.05)$. 


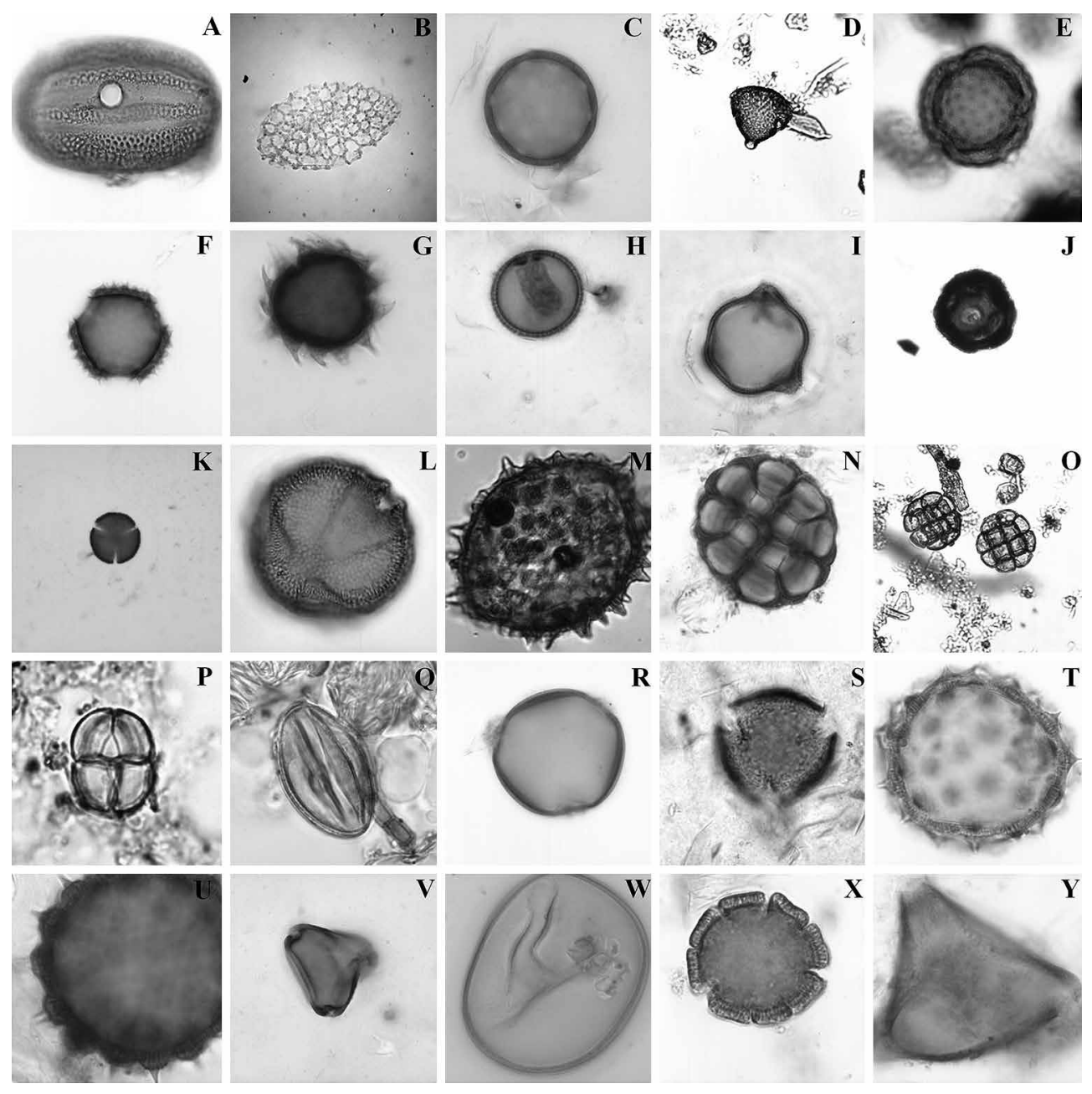

Fig. 1. Some pollen types found in Sonoran Desert propolis. Different scales are indicated for each pollen type. Acanthaceae: Dicliptera (A, 1000x). Agavaceae: Agave angustifolia (B, 400x). Chenopodiaceae-Amaranthaceae: Chenopodium sp. (C, 1000x), Malvaceae: Ceiba acuminata (D, 200x). Asteraceae: Ambrosia (E, 1000x), Asteraceae (F, 1000x), Asteraceae (G, 1000x). Burseraceae: Bursera laxiflora (H, 1000x), Bursera microphylla (I, 1000x). Cactaceae: Cylindropuntia thurberi (J, 200x), Stenocereus alamosensis (K, 200x). Fabaceae: Caesalpinia pulcherrima (L, 1000x). Convolvulaceae: Ipomoea arborescens (M, 400x). Fabaceae: Acacia sp. (N, 1000x), Havardia type (O, 400x), Mimosa distachya var. laxiflora (P, 1000x), Olneya tesota (Q, 1000x), Prosopis velutina (R, 1000x). Fagaceae: Quercus sp. (S, 1000x). Malvaceae: Abutilon (type, T, 1000x), Herissantia crispa (U, 1000x). Myrtaceae: Eucalyptus sp (V, 1000x). Poaceae sp. (W, 1000x). Rubiaceae: Mitracarpus hirtus (X, 1000x). Sapindaceae: Cardiospermum halicacabum (Y, 1000x). 
Vargas-Sánchez et al.: Pollen profile of propolis on the eastern edge of the Sonoran Desert

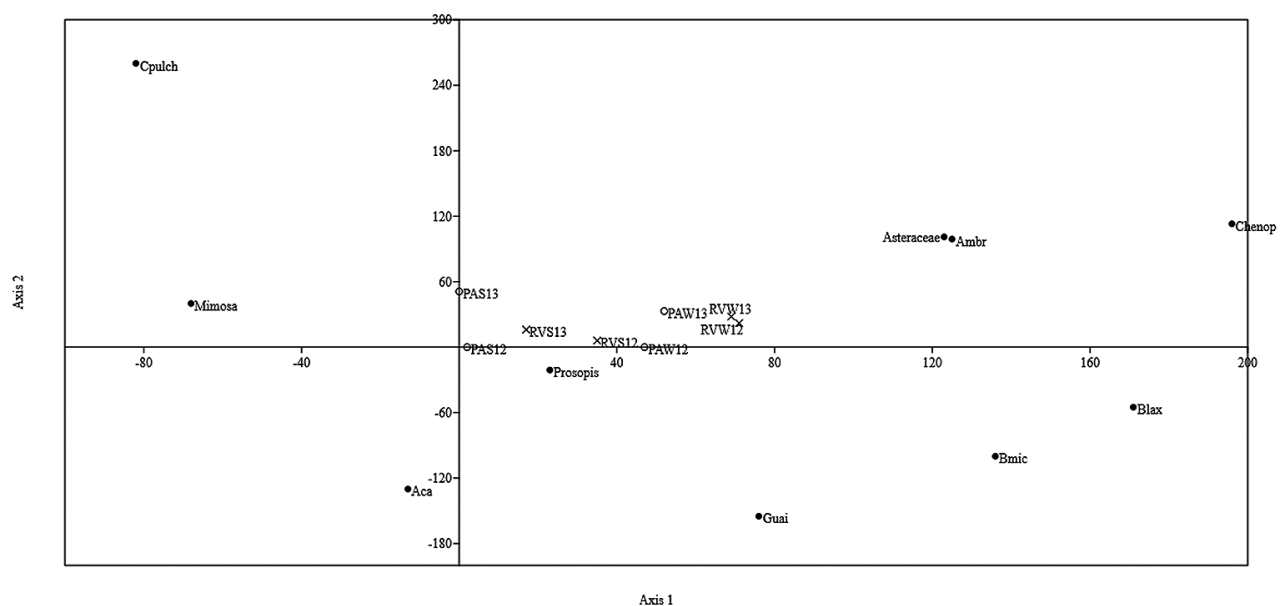

Fig. 2. Detrended correspondence analysis of propolis samples (A) and pollen taxa (B).

Prosopis in the riparian vegetation of RV). Additionally, Mimosa has more intense flowering periods in summer and Prosopis in the spring, although both plants flower from spring to late summer.

Statistical correlation analyses carried out in order to compare the relationship between the measured meteorological parameters (temperature and rainfall) and the most important pollen types (Mimosa distachya var. laxiflora and Prosopis velutina) showed significant association $(P<0.05)$ between temperature, rainfall and pollen content (Table 3).

Table 3. Correlation between the most representative propolis pollen taxa from central Sonora and values of average annual temperatures and rainfall.

\begin{tabular}{lcc}
\hline & Mimosa & Prosopis \\
\hline 2012 & & \\
Temperature & 0.483 & 0.976 \\
Rainfall & 0.809 & 0.915 \\
2013 & & \\
Temperature & 0.548 & 0.832 \\
Rainfall & 0.836 & 0.935 \\
\hline
\end{tabular}

Temperature in ${ }^{\circ} \mathrm{C}$. Rainfall in $\mathrm{mm}$. 


\section{DISCUSSION}

Sonoran Desert scrub and foothill thornscrub cover much of northwestern Mexico, with numerous trees belonging to the Fabaceae family (Robinson, 1898). Fabaceae is an important family of flowering plants known to be the most visited sources for honey, wax and propolis production in tropical regions (Almaraz-Abarca et al., 2007) and is made up of herbaceous plants, shrubs and trees, thereby significantly imprinting the landscape.

The genus Mimosa, which includes Mimosa distachya var. laxiflora (catclaw), is a plant of the Leguminosae (Fabaceae) Mimosoideae subfamily and is native to Northern and Southern America (Robinson, 1898). In Mexico it is mainly distributed in the region of northern Mexico corresponding to the states of Baja California, Chihuahua, Sinaloa and Sonora and in southern Mexico in Colima, Jalisco, Michoacán, Nayarit and Yucatán (Molina-Freaner et al., 2010; Instituto de Biología, 2010). Many species of mimosas are economically and socially important, as they are used for reforestation, timber production and medicinal purposes, as well as their ability to fix nitrogen. Flowers of several Mimosa species are also a major source of nectar for honey production (Simon et al., 2011). The genus Prosopis, which includes Prosopis velutina (mesquite), is also found within the family Fabaceae, subfamily Mimosoideae, and is native to the Asian, African and American continents. The genus includes 44 species distributed in arid and semiarid regions (Burkart, 1976; Palacios, 2006). In Mexico it has a wide distribution and is found along the Pacific coast, from the states of Michoacán to Oaxaca, in the Gulf of Mexico in Nuevo León, Tamaulipas and northern Veracruz and throughout the central region of the country (Gallegos-Infante et al., 2013). Mesquite is an important plant species due to its high potential for nitrogen fixation in very dry areas and during drought seasons, and it provides shelter and food to humans and many species of animals that feed on its leaves, fruits nectar and pollen (Almaraz-Abarca et al., 2007).

The pollen content in propolis samples can have different origins, and the frequency of the type of pollen can be indicative of the plant species used by honeybees to make the propolis (Silva et al., 2013). The results obtained for Sonoran Desert propolis showed that the most predominant pollen types belonged to the most abundant plant sources in the region (Asteraceae, Malvaceae, Cactaceae and Fabaceae families), and the minor pollen types were obtained from minor plant sources. In all propolis samples the most frequent pollen types were Mimosa distachya var. laxiflora and Prosopis velutina, which is in agreement with the central Sonoran honey studies carried out by Rivero-Montes (2000) and Burboa-Zazueta (2004). 
In another investigation, Mohamed and Afaf (2004) reported that the botanical origin of propolis samples from Sudan (Alfaki Hashim, Khartoum State and Al Abbasia, South Kordofan State) came from the following plant families: Mimosaceae, Malvaceae and Caesalpiniaceae. These were the most frequent pollen families (more than 50 grains were found) in Alfaki Hashim, and Mimosaceae was most abundant in the Al Abbasia region. Regarding propolis samples from Al Abbasia, the following, all belonging to the Fabaceae family, were considered to be dominant pollen sources (16-45\% of total content): Mimosa pigra, Mimosa sp. and Acacia sp. However, in other geographical regions, different taxa are identified in propolis samples. For example, Pulido-Avila et al. (1998) evaluated the pollen content of a propolis sample from Capilla de Milpillas and Telpatitlan de Morelos (Jalisco, Mexico) to evaluate which plants were used by bees to collect resins. Pollen analysis revealed a total of 30 pollen types, and Eucalyptus sp. (35.2\%) and Fraxinus sp. (19.9\%) were the main genera from which the honeybees obtained the resins. Other minor pollen types $(<15 \%)$ were Compositae (five pollen types), Quercus, Dicliptera, Bursera, Citrus, Mimosa and Ipomoea sp., among others. In the same way, Montenegro et al. (2000) revealed that the botanical origin of Chilean propolis is Eucalyptus. However, the Eucalyptus pollen frequencies $(<2 \%)$ reported in our work for the central Sonoran region do not reflect those of previous studies. In several regions of Brazil, Santos et al. (2011) established the plant origin of propolis samples, indicating that most representative pollen types for all samples were Schinus, Vernonia, Diclenia, Hyptis, Myrcia, Weinmannia, Baccharis and Eucalyptus. In contrast, Moreira et al. (2008) discovered an important contribution of different pollen components such as Pinus sp. (0-15\%), Castanea sativa (0-45\%), Populus tremula (30-50\%) and others (some of them with less than 5\%) in propolis from Portugal (Bornes and Fundão regions).

The above mentioned results show that bees produce propolis from surrounding vegetation, which includes different taxa according to the region, although similar ecosystems may provide taxonomically comparable propolis sources. Pollen content depends on plant diversity at a local and regional scale, and these studies confirm the importance of identifying the pollen content, geographical origin and collection season of propolis samples for their subsequent categorization and identification.

The slight statistically supported geographical separation of the samples was expected since the flora of both studied regions is similar. Otherwise, the high correlation found between propolis samples and their pollen content (floral origin) coincides with Barth and da Luz (2009), who found a strong correlation between propolis samples and pollen sources, which were considered to be the main characteristic 
component of propolis samples. Results have also shown that flowering species react differentially to temperature and water stress (meteorological conditions), which can subsequently affect the pollen composition (Rasmussen, 2002) of propolis, thereby explaining the yearly differences in propolis samples.

\section{CONCLUSIONS}

Six propolis samples were found to be bifloral (i.e. pollen types had secondary percentages, $15-45 \%)$, and two were multifloral $(<15 \%)$. The multifloral propolis samples were collected in winter. In total, 22 plant families and 42 species, or pollen types, were found in eight propolis samples. The Fabaceae family was dominant $(>45 \%)$ in both regions and during both seasons. Pollen from Mimosa distachya var. laxiflora and Prosopis velutina were the most representative, and this fact can be explained by the abundance of these plants in the thorn scrub foothills and desert scrub surrounding the beehives, as well as the high level of pollen production by these plants during the second harvesting period (summer), indicating that these are the most frequently visited by bees. The multivariate analysis was helpful in identifying the geographical and seasonal differentiation of the propolis samples, particularly those containing high frequencies of Mimosa and Prosopis pollen. In addition, the results of this study provide a list of plants from which the bees could obtain raw material to form propolis: these plants are the key to determining the geographical origin and characteristics of Sonoran propolis.

\section{ACKNOWLEDGMENTS}

We thank all the bee-keepers for allowing us to study their beehives. We also thank Livier Zavala-Cárdenas and Gerardo Reyna-Cañez for their technical support and academic assistance.

\section{LITERATURE CITED}

Almaraz-Abarca, N., M. da Graça-Campos, J. A. Ávila-Reyes, N. Naranjo-Jiménez, J. Herrera-Corral and L. González-Valdez. 2007. Antioxidant activity of polyphenolic extract of monofloral honeybee-collected pollen from mesquite (Prosopis juliflora, Leguminosae). J. Food Comp. Anal. 20: 119-124. 
Barth, O. M. 2004. Melissopalynology in Brazil: a review of pollen analysis of honeys, propolis and pollen loads of bees. Scientia Agric. 61: 342-350.

Barth, O. M. and C. F. P. da Luz. 2009. Palynological analysis of Brazilian red propolis samples. J. Apic. Res. 48: 181.

Brito-Castillo, L., M. A. Crimmins and S. C. C. Díaz. 2010. Clima. In: Molina-Freaner, F. E. and T. R. Van-Devender (eds.). Diversidad biológica de Sonora. Universidad Nacional Autónoma de México. México, D.F. Mexico. pp. 73-96.

Burboa-Zazueta, M. G. 2004. Identificación y caracterización de compuestos fenólicos presentes en la miel de mezquite (Prosopis sp.) y palo fierro (Olneya tesota): marcadores químicos del origen botánico. PhD thesis. Centro de Investigación en Alimentación y Desarrollo, A.C., Hermosillo, Mexico. 133 pp.

Burkart, A. 1976. A monograph of the genus Prosopis (Leguminosae subfam. Mimosoideae). J. Arnold Arbor. 57: 219-249.

Erdtman, G. 1960. The acetolysis method: A revised description. Svensk Bot. Tidskr. 54: 561-564.

Farré, R., I. Frasquet and A. Sánchez. 2004. El propolis y la salud. Ars. Pharma. 45: 21-43.

Felger, R. S., M. B. Johnson and M. F. Wilson. 2001. The trees of Sonora, Mexico. Oxford University Press. New York, USA. 400 pp.

Felger, R. S., B. T. Wilder and H. Romero-Morales. 2013. Plant life of a Desert Archipelago: Flora of the Sonoran Islands in the Gulf of California, Mexico. University of Arizona Press. Tucson, USA. 748 pp.

Gallegos-Infante, J. A., N. E. Rocha-Guzmán, R. F. González-Laredo and M. A. GarcíaCasas. 2013. Efecto del procesamiento térmico sobre la capacidad antioxidante de pinole a base de vainas de mezquite (Prosopis laevigata). CyTA-J. Food. 11: 162-170.

Hodgson, W. C. 2001. Food plants of the Sonoran Desert. University of Arizona Press. Tucson, USA. 313 pp.

INEGI. 2012. Clima. Instituto Nacional de Estadística y Geografía. Available in: http:// www.oeidrus-sonora.gob.mx/documentos/Geografia/HISTORIA\%20Y\%20GEO/ URES.pdf.

Instituto de Biología. 2010. Colecciones biológicas: "Mimosa distachya var. distachya IBUNAM: MEXU: PVsn1263”. UNIBIO: Colecciones Biológicas. Instituto de Biología, Universidad Nacional Autónoma de México. Available in: http://unibio.unam.mx/ collections/specimens/urn/IBUNAM:MEXU:PVsn1263. Accesed: 5 may 2015.

Martínez-Yrízar, A., R. S. Felger and A. Búrquez. 2010. Los ecosistemas terrestres: un diverso capital natural. In: Molina-Freaner, F. E. and T. R. Van-Devender (eds.). Diversidad biológica de Sonora. Universidad Nacional Autónoma de México. México, D.F, Mexico. pp. 129-156.

Matos, V. R., S. M. Alencar and F. A. R. Santos. 2014. Pollen types and levels of phenolic compounds in propolis produced by Apis mellifera L. (Apidae) in an area of the semiarid region of Bahia, Brazil. Anais Acad. Bras. Ci. 86: 407-418.

Mohamed, S. A. E. S. and M. E. S. Afaf. 2004. Botanical origin of the Sudanese propolis in central areas. University of Kentucky. J. Agric. Sci. 12: 9 pp.

Molina-Freaner, F. E. and T. R. Van-Devender (eds.). 2010. Diversidad biológica de Sonora. Universidad Nacional Autónoma de México. México, D.F. Mexico. 500 pp. 
Montenegro, G., B. Timmermann, R. Peña, A. Mujica and G. Ávila. 2000. Pollen grains and vegetative structures in propolis as indicators of potential drugs in Chilean plants. Int. J. Exp. Bot. 66: 15-23.

Montenegro, G., A. M. Mujica, R. C. Peña, M. Gómez, I. Serey and B. M. Timmermann. 2004. Similitude pattern and botanical origin of the Chilean propolis. Phyton 53: 145-153.

Moreira, L., L. G. Dias, J. A. Pereira and L. Estevinho. 2008. Antioxidant properties, total phenols and pollen analysis of propolis samples from Portugal. Food Chem. Tox. 46: 3482-3485.

Palacios, R. A. 2006. Los mezquites mexicanos: biodiversidad y distribución geográfica. Bol. Soc. Arg. Bot. 41(1-2): 99-121.

Peña, R. C. 2008. Propolis standardization: a chemical and biological review. Cienc. Inv. Agr. 35: 11-20.

Pulido-Ávila, M. G. and Jiménez-Reyes. 1998. Contenido de granos de polen de una muestra de propóleo. Boletín, IBUG 5(1-3): 493-504.

Rasmussen, A. 2002. The effects of climate change on the birch pollen season in Denmark. Aerobiol. 18: 253-265.

Rivero-Montes, L. K. 2000. Análisis físico-químico y caracterización polínica de miel de abeja en la región central de Sonora. BSc. Thesis. Universidad de Sonora. Hermosillo, Mexico. 126 pp.

Robinson, B. L. 1898. Revision of the North American and Mexican species of Mimosa. Contr. Gray Herb. Harvard Univ. 13: 305-331.

Roubik, D. W. and Moreno, P. 1991. Pollen and spores of Barro Colorado Island [Panama]. Monographs in Systematic Botany from the Missouri Botanical Garden, 36..Missouri Botanical Garden. St. Louis, MO. USA. iv +270 pp.

Sánchez-Escalante, A., R. D. Vargas-Sánchez, M. Valenzuela, M. A. de la Rosa, G. Torrescano and J. P. Camou. 2009. Evaluation of antioxidant activity of propolis produced in northwest of Mexico for fresh beef patties. Presented at 2009 Annual Meeting of the Institute of Food Technologists. Book abstract 027-31. Anaheim, USA.

Santos, F. A. R. 2011. Identificação botânica do pólen apícola. Magistra 23: 5-9.

SEINet. 2015. Pollen profile of Sonoran Desert propolis produced in central Sonora, Mexico. Hermosillo, Mexico. Available in: http://swbiodiversity.org/seinet/checklists/ checklist.php? cl=3497\&emode=0. Accessed: 5 may 2015 .

Servicio Meteorológico Nacional. 2012. Reporte del clima en México 2012. Servicio Meteorológico Nacional. México, D.F., Mexico. 23 pp.

Servicio Meteorológico Nacional. 2013. Reporte del clima en México 2013. Servicio Meteorológico Nacional. México, D.F., Mexico. 23 pp.

Silva, C. R. B. D., T. Putarov and R. D. O Orsi. 2013. Pollen spectrum of propolis samples from São Paulo State, Brazil. Acta Scientiarum. Anim. Sci. 35: 297-300.

Simon, M. F., R. Grether, L. P. de Queiroz, T. E. Särkinen, V. F. Dutra and C. E. Hughes. 2011. The evolutionary history of Mimosa (Leguminosae): toward a phylogeny of the sensitive plants. Amer. J. Bot. 98: 1201-1221.

SPSS Statistics. 2012. Statistical Package for the Social Sciences. SPSS Statistics. Chicago, USA. 
Turner, R. M., J. E. Bowers and T. L. Burgess. 2005. Sonoran Desert plants: an ecological atlas. University of Arizona Press, Tucson, USA. 504 pp.

Vargas-Sánchez, R. D., G. R. Torrescano-Urrutia and A. Sánchez-Escalante. 2013. El propóleos: conservador potencial para la industria alimentaria. Interciencia 38: 705711.

Vargas-Sánchez, R. D., G. R. Torrescano-Urrutia, A. M. Mendoza-Wilson, B. VallejoGalland, E. Acedo-Félix, J. J. Sánchez-Escalante, M. C. Peñalba-Garmendia and A. Sánchez-Escalante. 2014. Mechanisms involved in antioxidant and antibacterial activity of propolis. Biotecnia 16: 32-37.

Velázquez, C., M. Navarro, A. Acosta, A. Angulo, Z. Domínguez, R. Robles, R. RoblesZepeda, E. Lugo, F. M. Goycoolea, E. F. Velázquez, H. Astiazaran and J. Hernández. 2007. Antibacterial and free-radical scavenging activities of Sonoran propolis. J. Appl. Microbiol. 103: 1747-1756.

Warakomska, Z. and W. Maciejewicz. 1992. Microscopic analysis of propolis from Polish regions. Apidol. 23: 277-283. 